\title{
Correlation matrices, Clifford algebras, and completely positive semidefinite rank
}

\author{
Anupam Prakash* and Antonios Varvitsiotis ${ }^{\dagger}$ \\ Nanyang Technological University, Singapore and Centre for Quantum \\ Technologies, Singapore
}

October 1, 2018

\begin{abstract}
A symmetric $n \times n$ matrix $X$ is completely positive semidefinite (cpsd) if there exist $d \times d$ positive semidefinite matrices $\left\{P_{i}\right\}_{i=1}^{n}$ (for some $d \in \mathbb{N}$ ) such that $X_{i j}=\operatorname{Tr}\left(P_{i} P_{j}\right)$, for all $i, j \in\{1, \ldots, n\}$. The cpsd-rank of a cpsd matrix is the smallest $d \in \mathbb{N}$ for which such a representation is possible. It was shown independently in [19] and [12] that there exist completely positive semidefinite matrices with sub-exponential cpsd-rank. Both proofs were obtained using fundamental results from the quantum information literature as a black-box. In this work we give a self-contained and succinct proof of the existence of completely positive semidefinite matrices with sub-exponential cpsd-rank. For this, we introduce matrix valued Gram decompositions for correlation matrices and show that for extremal correlations, the matrices in such a factorization generate a Clifford algebra. Lastly, we show that this fact underlies and generalizes Tsirelson's results concerning the structure of quantum representations for extremal quantum correlation matrices.
\end{abstract}

\section{Introduction}

A symmetric $n \times n$ matrix $X$ is completely positive semidefinite (cpsd) if there exist $d \times d$ Hermitian positive semidefinite matrices $\left\{P_{i}\right\}_{i=1}^{n}$ (for some integer $d \geq 1$ ) satisfying

$$
X_{i j}=\operatorname{Tr}\left(P_{i} P_{j}\right) \text {, for all } i, j \in[n] .
$$

The set of $n \times n$ cpsd matrices forms a convex cone denoted by $\mathcal{C S}_{+}^{n}$. The cpsd cone was introduced recently to provide linear conic formulations for the quantum analogues of various classical graph parameters [14, 20]. Subsuming these results, it was shown in [21] that the set of joint probability distributions that can be generated using quantum resources can be expressed as the projection of an affine section of the $\mathcal{C S}_{+}^{n}$ cone.

\footnotetext{
*Email: aprakash@ntu.edu.sg

†Email: avarvits@gmail.com
} 
The completely positive semidefinite rank of $X \in \mathcal{C S}_{+}^{n}$, denoted by cpsd-rank $(X)$, is defined as the least $d \geq 1$ for which there exist $d \times d$ Hermitian positive semidefinite matrices $P_{1}, \ldots, P_{n}$ satisfying $X_{i j}=\operatorname{Tr}\left(P_{i} P_{j}\right)$, for all $i, j=1, \ldots, n$. The study of the cpsd-rank also has strong physical motivation as it captures the size of a quantum system that is necessary to generate a quantum probability distribution [19].

Besides their physical motivation, the cpsd cone and the cpsd-rank are also interesting from the perspective of linear conic optimization. Firstly, the cpsd cone and the cpsdrank are non-commutative analogues of the completely positive cone and the cp-rank. A symmetric $n \times n$ matrix $X$ is completely positive (cp) if there exist vectors $\left\{p_{i}\right\}_{i=1}^{n} \subseteq \mathbb{R}_{+}^{d}$ (for some $d \geq 1$ ) such that $X_{i j}=p_{i}^{\top} p_{j}$, for all $i, j=1, \ldots, n$. The $c p$-rank of a cp matrix $X$ is the least integer $d \geq 1$ for which such a factorization is possible. The cp cone has been extensively studied as any quadratic program with a mix of binary and continuous variables can be formulated as a linear conic program over the cp cone [5].

Secondly, cpsd factorizations correspond to symmetric psd-factorizations. Recall that a $d$-dimensional psd factorization of a matrix $X \in \mathbb{R}_{+}^{n \times m}$ consists of two families of $d \times d$ Hermitian positive semidefinite matrices $\left\{P_{i}\right\}_{i=1}^{n},\left\{Q_{j}\right\}_{j=1}^{m}$ satisfying $X_{i j}=\operatorname{Tr}\left(P_{i} Q_{j}\right)$ for all $i=1, \ldots, n$ and $j=1, \ldots, m$. The $p s d$-rank of an entrywise nonnegative matrix $X$ is the smallest dimension of a psd factorization. The psd-rank has been extensively studied as it characterizes the semidefinite extension complexity of polytopes and as a result, captures the efficacy of semidefinite programming for hard combinatorial problems [9].

Combining Carathéodory's Theorem (e.g. see [3, Theorem 1.34]) with the atomic reformulation of the cp-rank we have that $\operatorname{cp-rank}(X) \leq\left(\begin{array}{c}n+1 \\ 2\end{array}\right)$ for any $X \in \mathcal{C P}^{n}$. The best upper bound currently is $\frac{n^{2}}{2}+O\left(n^{3 / 2}\right)$ [4], which is asymptotically tight with respect to the Drew-Johnson-Loewy lower bound of $\left|n^{2} / 4\right|$, for $n \geq 4$ [7]. Furthermore, the psd-rank of a matrix $X \in \mathbb{R}_{+}^{n \times m}$ is always at most $\min \{n, m\}$ as there is a factorization $X_{i j}=\operatorname{Tr}\left(P_{i} Q_{j}\right)$ for diagonal matrices $P_{i}=\operatorname{diag}\left(e_{i}\right)$ and $Q=\operatorname{diag}\left(X^{j}\right)$ where $e_{i}, i \in[n]$ are the standard basis vectors for $\mathbb{R}^{n}$ and $X^{j}$ is the $j$-th column of $X$.

In contrast to these related notions of matrix ranks, it was shown independently in [19] and [12], that there exist cpsd matrices whose cpsd-rank is sub-exponential in terms of their dimension. Specifically, we have that:

Theorem 1.1 ([19, 12]). For any $n \in \mathbb{N}$ there exists a matrix $X_{n} \in \mathcal{C S}_{+}^{2 n}$ such that

$$
\operatorname{cpsd}-\operatorname{rank}\left(X_{n}\right) \geq 2^{\left\lfloor r_{\max }(n) / 2\right\rfloor},
$$

where $r_{\max }(n)$ is the greatest integer satisfying $\left(\begin{array}{c}r+1 \\ 2\end{array}\right) \leq n$, i.e.,

$$
r_{\max }(n)=\left\lfloor\frac{\sqrt{1+8 n}-1}{2}\right\rfloor .
$$

Both proofs of Theorem 1.1 [19, 12] were obtained using fundamental results from the quantum information literature as a black-box [23]. Our main goal in this article is to give a self-contained proof of Theorem 1.1 that bypasses the quantum information results that were used in the original proofs [19, 12], and as such, makes it accessible to the broader mathematical community. Furthermore, the new proof presented in this article highlights certain matrix factorizations of the elliptope as the main underlying mathematical tool and paves the way for further generalizations. 
Main technical result. The correlation matrix of the random variables $X_{1}, \ldots, X_{n}$ is the $n \times n$ matrix whose $(i, j)$ entry is equal to the correlation between $X_{i}$ and $X_{j}$, i.e.,

$$
\mathbb{E}\left[\left(X_{i}-\mu_{i}\right)\left(X_{j}-\mu_{j}\right)\right] / \sigma_{i} \sigma_{j},
$$

where $\mu_{i}, \sigma_{i}$ denote the mean and standard deviation of $X_{i}$. Correlation matrices capture the association between random variables and their use is ubiquitous in statistics.

It is easy to verify that correlation matrices are positive semidefinite and have all diagonal entries equal to one. Conversely, any such matrix can be expressed as a correlation matrix for some family of random variables. Thus, the set of $n \times n$ correlation matrices coincides with the $n$-dimensional elliptope, denoted by $\mathcal{E}_{n}$, defined as the set of $n \times n$ symmetric positive semidefinite matrices with diagonal entries equal to one, i.e.,

$$
\mathcal{E}_{n}:=\left\{E \succeq 0: E_{i i}=1(i \in[n])\right\} .
$$

The elliptope is a spectrahedral set whose structure has been extensively studied (e.g. see [6] and references therein). Its significance is illustrated by the fact that it corresponds to the feasible region of various semidefinite programs that are used to approximate NPhard combinatorial optimization problems (e.g. MAX-CUT [10]).

In this work we introduce and study matrix factorizations of a specific form for correlation matrices. Informally, our main result is that for extreme points of the set of correlation matrices, the matrices in such a factorization generate a Clifford algebra.

Recall that the rank- $n$ Clifford algebra, denoted by $\mathcal{C}_{n}$, is the universal $C^{*}$-algebra generated by Hermitian indeterminates $z_{1}, \ldots, z_{n}$ satisfying the following relations:

$$
z_{i} z_{j}+z_{j} z_{i}=2 \delta_{i j} I, \quad \text { for all } i, j \in[n] .
$$

Furthermore, it is well-known that depending on the parity of $n$, the algebra $\mathcal{C}_{n}$ has either one or two irreducible representations, each of dimension $2^{\lfloor n / 2\rfloor}$, e.g., see [10, Chapter 6].

Having introduced Clifford algebras, we now formally state our main technical result.

Theorem 1.2. Let $E$ be an extreme point of $\mathcal{E}_{k}$ with $\operatorname{rank}(E)=n$ and let $A$ be a fullrank principal submatrix of $E$. Assume that $E=\left(\begin{array}{c}A \\ C^{\top}\end{array}{ }_{B}^{C}\right)$ and consider $d \times d$ Hermitian matrices $\left\{X_{i}\right\}_{i=1}^{n},\left\{Y_{j}\right\}_{j=1}^{k-n}, K$ satisfying

(i) $E=\operatorname{Gram}\left(K X_{1}, \ldots, K X_{n}, Y_{1} K, \ldots, Y_{k-n} K\right)$;

(ii) $X_{i}^{2}=Y_{j}^{2}=I_{d}$, for all $i \in[n], j \in[k-n]$;

(iii) $\operatorname{Tr}\left(K^{2}\right)=1$ and $K$ is positive definite.

Then, the algebra $\mathbb{C}\left[X_{1}, \ldots, X_{n}\right]$ is isomorphic to the rank-n Clifford algebra $\mathcal{C}_{n}$. In particular, the size of the matrices $X_{1}, \ldots, X_{n}$ is lower bounded by $2^{\lfloor n / 2\rfloor}$.

The proof of Theorem 1.2 is given in Section 1.2. Throughout this work, we refer to any family of matrices $\left\{X_{i}\right\}_{i=1}^{n},\left\{Y_{j}\right\}_{j=1}^{k-n}, K$ satisfying conditions $(i),(i i)$ and (iii) above as a matrix factorization of the correlation matrix $E$.

A couple of comments are in order concerning Theorem 1.2. First, it is well-known that there exists a rank- $n$ extreme point of $\mathcal{E}_{k}$ if and only if $k \geq\left(\begin{array}{l}n \\ 2\end{array}\right)$ (e.g. see Section 2.1), and this condition will be satisfied whenever we apply Theorem 1.2. Second, although not immediately obvious, we show in Lemma 2.6 that any correlation matrix admits such a matrix factorization (where we can even always take $K$ to be a multiple of the identity). Third, it is worth noting that Theorem 1.2 remains valid when $(i)$ is replaced with: 
$\left(i^{\prime}\right) E=\operatorname{Gram}\left(K X_{1}, \ldots, K X_{n}, K Y_{1}, \ldots, K Y_{k-n}\right)$.

and also when $(i),(i i)$ and $(i i i)$ are replaced with:

$\left(i^{\prime \prime}\right) E=\operatorname{Gram}\left(A_{1}, \ldots, A_{n}, B_{1}, \ldots, B_{k-n}\right) ;$

$\left(i i^{\prime \prime}\right) A_{i}^{2}=B_{j}^{2}=\frac{1}{d} I_{d}$, for all $i \in[n], j \in[k-n]$.

Using Theorem 1.2, in Section 3 we give a short proof of Theorem 1.1.

Related work from quantum information theory. The image of the elliptope $\mathcal{E}_{n+m}$ under the projection operator

$$
\pi: \mathcal{S}_{n+m} \rightarrow \mathbb{R}^{n \times m}, \quad\left(\begin{array}{cc}
A & C \\
C^{\top} & B
\end{array}\right) \mapsto C
$$

is known as the set of $(n, m)$ bipartite correlation matrices and is of central importance to quantum information theory. The link between quantum information theory and bipartite correlation matrices is best explained within the following framework, known in the physics literature as a Bell scenario [1]. Consider two parties, Alice and Bob, that share a bipartite quantum system, e.g., a pair of spin- $1 / 2$ particles. According to the postulates of quantum mechanics, the state of a bipartite quantum system with local dimension $d$ is described by a Hermitian psd matrix $\rho$ acting on $\mathbb{C}^{d} \otimes \mathbb{C}^{d}$ with $\operatorname{Tr}(\rho)=1$.

Independently and simultaneously, Alice and Bob measure their part of the system and then announce the outcomes of their measurements. By the postulates of quantum mechanics, the process of measuring a $d$-dimensional quantum system is described by a Hermitian matrix $H \in \mathbb{C}^{d \times d}$, called an observable. By the spectral theorem, for all observables $H$ we have the decomposition $H=\sum_{i=1}^{k} \lambda_{i} P_{i}$, where $\left\{\lambda_{i}\right\}_{i=1}^{k}(k \leq d)$ are the eigenvalues of $H$ and $\left\{P_{i}\right\}_{i=1}^{k}$ are the projectors onto the corresponding eigenspaces. The measurement defined by $H$ has possible outcomes $\left\{\lambda_{i}\right\}_{i=1}^{k}$. Upon measuring a state $\rho$, the outcome $\lambda_{i}$ is observed with probability $\operatorname{Tr}\left(\rho P_{i}\right)$. As a consequence, $\operatorname{Tr}(\rho H)$ is the expectation of the outcome upon performing the measurement $H$ on state $\rho$.

Throughout this paper, we only consider observables $H$ whose spectrum lies in $[-1,1]$, i.e., observables satisfying $H^{2} \preceq I$. Furthermore, we assume throughout that Alice has $n$ measurement choices given by the observables $M_{1}, \ldots, M_{n}$ and Bob has $m$ measurement choices denoted by $N_{1}, \ldots, N_{m}$. Accordingly, if their shared system is in state $\rho$ and Alice and Bob perform measurements $M_{i}$ and $N_{j}$ respectively, the expectation of the product of their answers is given by $\operatorname{Tr}\left(\rho M_{i} \otimes N_{j}\right)$. The $n \times n$ matrix whose $(i, j)$ entry is given by $\operatorname{Tr}\left(\rho M_{i} \otimes N_{j}\right)$ is called a quantum correlation matrix, cf. Definition 4.2.

Tsirelson showed in [23] that, in the two-outcome case, the set of quantum correlation matrices coincides with the set of bipartite correlation matrices ( $c f$. Theorem 4.1). This characterization has found numerous applications. As one example, this implies that one can optimize a linear function over the set of quantum correlation matrices (up to arbitrary precision) in polynomial-time, using semidefinite programming.

Additionally, Tsirelson studied the structural properties of the quantum state and the observables that are necessary to generate an extremal quantum correlation matrix. Roughly speaking, he showed that any quantum correlation matrix can be generated using observables $\left\{M_{i}\right\}_{i}$ and $\left\{N_{j}\right\}_{j}$, where all the anti-commutators $M_{i} M_{j}+M_{j} M_{i}$ are 
scalar multiples of the identity (and analogously for the $N_{j}$ 's). Moreover, he showed that for extremal quantum correlation matrices, such representations are essentially the only possible ones, e.g. see [23, Theorem 3.1] and [24, Theorem 3.8]. Using standard results concerning the representations of Clifford algebras, Tsirelson's work implies that the local dimension of a quantum system necessary to generate an extreme quantum correlation matrix can be lower bounded in terms of its rank.

Theorem 1.3 ([23, 24]). Given an extreme bipartite correlation matrix $C \in \operatorname{ext}\left(\pi\left(\mathcal{E}_{n+m}\right)\right)$, the local dimension of any tensor product representation is lower bounded by $2^{\lfloor\operatorname{rank}(C) / 2\rfloor}$.

Using Theorem 1.2, in Section 4 we derive Theorem 1.3. For this we show that any extreme quantum correlation matrix can be completed in a unique way to a correlation matrix and also, this completion is an extreme correlation matrix. Thus, lower bounds on matrix factorizations of extreme correlation matrices (such as Theorem 1.2) imply lower bounds on factorizations of extremal quantum correlation matrices (such as Theorem 1.3).

The proof of Theorem 1.2 relies on ideas from Tsirelson's work. Nevertheless, Theorem 1.2 strictly generalizes Theorem 1.3 as the projection of an extreme point is not necessarily extreme, i.e., there exist matrices $\left(\begin{array}{cc}A & C \\ C^{\top}\end{array}\right) \in \operatorname{ext}\left(\mathcal{E}_{n+m}\right)$ for which $C \notin \operatorname{ext}\left(\pi\left(\mathcal{E}_{n+m}\right)\right)$. A concrete example of such a matrix is given at the end of Section 4 .

Further related work and open problems. Theorem 1.2 provides an alternative interpretation of Tsirelson's results, by highlighting matrix factorizations of the elliptope as the underlying mathematical object. On the other hand, representations of generalized Clifford algebras (associated with a real zero polynomial) are related to the existence of determinantal representations of (powers of) hyperbolic polynomials, e.g. see [17, 18]. It is an interesting question whether representations of generalized Clifford algebras are related in a similar manner to other spectrahedra, more general than the elliptope.

Let $\mathcal{K}=\left\{K_{d}\right\}_{d}$ be a family of cones that is closed under direct sums and define

$$
\operatorname{Gram}(n, \mathcal{K})=\left\{\operatorname{Gram}\left(u_{1}, \ldots, u_{n}\right): u_{1}, \ldots, u_{n} \in K_{d}, \text { for some } d \in \mathbb{N}\right\},
$$

which is itself a convex cone. For a matrix $X \in \operatorname{Gram}(n, \mathcal{K})$ define $\operatorname{Gram-rank}(X)$ to be the least $d \geq 1$ such that $X$ can be realized as a Gram matrix of vectors in $K_{d}$. As noted earlier, for a matrix $X$ in $\operatorname{Gram}\left(n,\left\{\mathbb{R}^{d}\right\}_{d}\right)=\mathcal{S}_{+}^{n}$ and $\operatorname{Gram}\left(n,\left\{\mathbb{R}_{+}^{d}\right\}_{d}\right)=\mathcal{C P}_{n}$, the Gram-rank of $X$ is upper bounded by a polynomial in the dimension $n$ while for $\operatorname{Gram}\left(n,\left\{\mathcal{S}_{+}^{d}\right\}_{d}\right)=\mathcal{C S}_{+}^{n}$, Theorem 1.1 establishes a sub-exponential lower bound.

An interesting research direction is to prove upper and lower bounds for $\operatorname{Gram-\operatorname {rank}}(X)$ in terms of the dimension for matrices $X \in \operatorname{Gram}(n, \mathcal{K})$ for other families of cones and also, to investigate the cases for which there is a super polynomial lower bound. The closely related notion of generalized completely positive cones has been studied in [11].

Lastly, we mention that following the completion of this work, there have been exciting new results concerning the closedness of the set of quantum behaviors that imply the existence of completely positive semidefinite matrices (of fixed size) with arbitrarily large cpsd-rank [22, 8, 16].

\section{$1.1 \quad$ Preliminaries}

In this section we introduce the most important definitions, notation and background material that we use throughout this paper. 
Linear Algebra. Throughout, we use the shorthand notation $\left\{x_{i}\right\}_{i=1}^{n}:=\left\{x_{1}, \ldots, x_{n}\right\}$ and $[n]:=\{1, \ldots, n\}$. We denote by $\left\{e_{i}\right\}_{i=1}^{n}$ the standard basis of $\mathbb{C}^{n}$. The canonical inner product of two vectors $x, y \in \mathbb{R}^{n}$ is denoted by $\langle x, y\rangle$. We write $\operatorname{span}\left(\left\{x_{i}\right\}_{i=1}^{n}\right)$ for the linear span of the vectors $\left\{x_{i}\right\}_{i=1}^{n}$.

We denote by $\mathcal{M}_{d}$ the set of $d \times d$ complex matrices and by $\mathcal{H}_{d}$ (resp. $\mathcal{S}_{d}$ ) the set of $d \times d$ Hermitian (resp. symmetric) matrices. Given a matrix $X \in \mathcal{M}_{d}$, its transpose is denoted by $X^{\top}$ and its conjugate transpose by $X^{*}$. Furthermore, we denote by $X \otimes Y$ the Kronecker product of $X$ and $Y$. Throughout, we equip $\mathcal{H}_{d}$ with the Hilbert-Schmidt inner product $\langle X, Y\rangle:=\operatorname{Tr}\left(X Y^{*}\right)$. For a block matrix $X=\left(\begin{array}{c}A \\ C^{\top}\end{array}{ }_{B}^{C}\right) \in \mathcal{S}_{n+m}$ we use that

$$
\operatorname{rank}(X)=\operatorname{rank}(A) \Longleftrightarrow \exists n \times m \text { matrix } \Lambda \text { such that } C=A \Lambda \text { and } B=\Lambda^{\top} A \Lambda \text {. }
$$

A matrix $X \in \mathcal{H}_{d}$ is called positive semidefinite (psd) if $\psi^{*} X \psi \geq 0$, for all $\psi \in \mathbb{C}^{d}$. The set of $d \times d$ Hermitian psd (resp. symmetric psd) matrices forms a closed convex cone denoted by $\mathcal{H}_{+}^{d}\left(\right.$ resp. $\left.\mathcal{S}_{+}^{d}\right)$. We sometimes also write $X \succeq 0$ to indicate that $X$ is psd.

The Gram matrix of a family of vectors $\left\{x_{i}\right\}_{i=1}^{n} \subseteq \mathbb{R}^{d}$, denoted by $\operatorname{Gram}\left(x_{1}, \ldots, x_{n}\right)$ or $\operatorname{Gram}\left(\left\{x_{i}\right\}_{i=1}^{n}\right)$, is the symmetric $n \times n$ matrix whose $(i, j)$ entry is given by $\left\langle x_{i}, x_{j}\right\rangle$, for all $i, j \in[n]$. It is easy to see that an $n \times n$ matrix $X$ is positive semidefinite if and only if there exist vectors $x_{1}, \ldots, x_{n} \in \mathbb{R}^{k}$ (for some $k \geq 1$ ) such that $X=\operatorname{Gram}\left(x_{1}, \ldots, x_{n}\right)$. For any Gram matrix we have that $\operatorname{rank}\left(\operatorname{Gram}\left(\left\{x_{i}\right\}_{i=1}^{n}\right)\right)=\operatorname{dim}\left(\operatorname{span}\left(\left\{x_{i}\right\}_{i=1}^{n}\right)\right)$. Lastly, if $X=\operatorname{Gram}\left(x_{1}, \ldots, x_{n}\right)$ we make use the following property:

$$
\text { For any } \lambda \in \mathbb{R}^{n}: X \lambda=0 \Longleftrightarrow \sum_{i=1}^{n} x_{i} \lambda_{i}=0 \text {. }
$$

We use a well-known correspondence between $\mathcal{M}_{d}$ and $\mathbb{C}^{d} \otimes \mathbb{C}^{d}$ given by the map vec : $\mathcal{M}_{d} \rightarrow \mathbb{C}^{d} \otimes \mathbb{C}^{d}$, which is given by $\operatorname{vec}\left(e_{i} e_{j}^{*}\right)=e_{i} \otimes e_{j}$, on basis vectors and is extended linearly. The $\operatorname{vec}(\cdot)$ map is an isometry, i.e., $\langle X, Y\rangle=\langle\operatorname{vec}(Y)$, $\operatorname{vec}(X)\rangle$ for all $X, Y \in \mathcal{M}_{d}$. We also need the following fact:

$$
\operatorname{vec}(W)^{*}(X \otimes Y) \operatorname{vec}(Z)=\operatorname{vec}(W)^{*} \operatorname{vec}\left(X Z Y^{\top}\right)=\left\langle W, X Z Y^{\top}\right\rangle,
$$

Any vector $\psi \in \mathbb{C}^{d} \otimes \mathbb{C}^{d}$ can be uniquely expressed as $\psi=\sum_{i=1}^{d} \lambda_{i} y_{i} \otimes x_{i}$ for some integer $d \geq 1$, positive scalars $\left\{\lambda_{i}\right\}_{i=1}^{d}$, and orthonormal sets $\left\{y_{i}\right\}_{i=1}^{d} \subseteq \mathbb{C}^{d}$ and $\left\{x_{i}\right\}_{i=1}^{d} \subseteq \mathbb{C}^{d}$. An expression of this form is known as a Schmidt decomposition for $\psi$ and is derived by the singular value decomposition of $\operatorname{vec}^{-1}(\psi)$. Note that if $\psi=\sum_{i=1}^{d} \lambda_{i} y_{i} \otimes x_{i}$ is a Schmidt decomposition for $\psi$, then we have that $\|\psi\|_{2}^{2}=\sum_{i=1}^{d} \lambda_{i}^{2}$.

The Pauli matrices are given by

$$
I_{2}:=\left(\begin{array}{ll}
1 & 0 \\
0 & 1
\end{array}\right), X:=\left(\begin{array}{ll}
0 & 1 \\
1 & 0
\end{array}\right), Y:=\left(\begin{array}{cc}
0 & -i \\
i & 0
\end{array}\right), \text { and } Z:=\left(\begin{array}{cc}
1 & 0 \\
0 & -1
\end{array}\right) .
$$

Note that the (non-identity) Pauli matrices are Hermitian, their trace is equal to zero, they have \pm 1 eigenvalues and they pairwise anti-commute.

Clifford algebras. Throughout this section set $d:=2^{\lfloor r / 2\rfloor}$. It is well-known that

$$
\mathcal{C}_{r} \cong \mathcal{M}_{d}, \text { for even } r \text {, and } \mathcal{C}_{r} \cong \mathcal{M}_{d} \oplus \mathcal{M}_{d}, \text { for odd } r
$$


For a proof of this fact and additional details the reader is referred to [10, Chapter 6]. An explicit representation of $\mathcal{C}_{r}$ is obtained using the Brauer-Weyl matrices. Specifically, for $r=2 \ell$, the map $\gamma_{r}: \mathcal{C}_{r} \rightarrow \mathcal{H}_{d}$ given by

$$
\gamma_{r}\left(z_{i}\right)=Z^{\otimes(i-1)} \otimes X \otimes I_{2}^{\otimes(\ell-i)} \in \mathcal{H}_{d},(i \in[\ell]),
$$

and

$$
\gamma_{r}\left(z_{i+\ell}\right)=Z^{\otimes(i-1)} \otimes Y \otimes I_{2}^{\otimes(\ell-i)} \in \mathcal{H}_{d}, \quad(i \in[\ell]) .
$$

is a complex representation of $\mathcal{C}_{r}$. Furthermore, in the case where $r=2 \ell+1$ we define $\left\{\gamma_{r}\left(z_{i}\right)\right\}_{i=1}^{2 \ell}$ as described in (9) and (10) and additionally set $\gamma_{r}\left(z_{2 \ell+1}\right)=Z^{\otimes \ell}$.

Lastly, we collect some properties of the map $\gamma_{r}$ which will be crucial for our results in the next section. Specifically, setting $d=2^{\lfloor r / 2\rfloor}$, for all $x, y \in \mathbb{R}^{n}$ we have the following:

$$
\gamma_{r}(x)^{2}=\|x\|^{2} I_{d} \quad \text { and } \quad d\langle x, y\rangle=\operatorname{Tr}\left(\gamma_{r}(x) \gamma_{r}(y)\right) .
$$

Convexity. A set $C \subseteq \mathbb{R}^{n}$ is convex if for all $a, b \in C$ and $\lambda \in[0,1]$ we have that $\lambda a+(1-\lambda) b \in C$. A subset $F \subseteq C$ is called a face of $C$ if $\lambda c_{1}+(1-\lambda) c_{2} \in F$ implies that $c_{1}, c_{2} \in F$, for all $c_{1}, c_{2} \in C$ and $\lambda \in[0,1]$. We say that $c$ is an extreme point of the convex set $C$ if the singleton $\{c\}$ is a face of $C$. We denote by $\operatorname{ext}(C)$ the set of extreme points of the convex set $C$. By the Krein-Milman theorem, any compact convex subset of $\mathbb{R}^{n}$ is equal to the convex hull of its extreme points, e.g. see [2].

\section{Correlation matrices, extreme points, and matrix factorizations}

\subsection{Extreme correlation matrices and quadratic maps}

An operator valued quadratic map is a function $Q: \mathbb{R}^{r} \rightarrow \mathcal{H}_{d}$ (for some $d \geq 1$ ) such that $Q(a x)=a^{2} Q(x)$, for all $x \in \mathbb{R}^{r}$ and $a \in \mathbb{R}$. The following result from [23] will be crucial.

Lemma 2.1. Consider vectors $\left\{u_{i}\right\}_{i=1}^{n} \subseteq \mathbb{R}^{r}$ satisfying $\operatorname{span}\left(u_{1}, \ldots, u_{n}\right)=\mathbb{R}^{r}$ and

$$
\operatorname{span}\left(u_{1} u_{1}^{\top}, \ldots, u_{n} u_{n}^{\top}\right)=\mathcal{S}_{r} .
$$

Then, for any operator valued quadratic map $Q: \mathbb{R}^{r} \rightarrow \mathcal{H}_{d}$ we have that

$$
Q\left(u_{i}\right)=0, \text { for all } i \in[n] \Longrightarrow Q=0 .
$$

Proof. First consider the case $d=1$, i.e., we have a quadratic form $Q: \mathbb{R}^{r} \rightarrow \mathbb{R}$. Let $M_{Q} \in \mathcal{S}_{r}$ be the symmetric matrix, corresponding to the bilinear form associated to $Q$, with respect to the standard basis $\left\{e_{i}\right\}_{i=1}^{r}$ of $\mathbb{R}^{r}$. By assumption (13) we have that $Q\left(u_{i}\right)=u_{i}^{\top} M_{Q} u_{i}=0$ for all $i \in[n]$ and thus, (12) implies that $M_{Q}=0$. For the case $d>1$, since $\mathcal{H}_{d}$ is a $d^{2}$-dimensional vector space over the real numbers, we can equivalently view $Q$ as a quadratic form $Q: \mathbb{R}^{r} \rightarrow \mathbb{R}^{d^{2}}$ where $x \mapsto\left(Q_{1}(x), \ldots, Q_{d^{2}}(x)\right)$. As all the $Q_{i}$ 's are real valued quadratic forms, the proof is concluded from the base case.

Next, we recall the following well-known characterization of the extreme points of $\mathcal{E}_{n}$. 
Theorem $2.2([15])$. Consider $E \in \mathcal{E}_{n}$ with $r=\operatorname{rank}(E)$ and let $E=\operatorname{Gram}\left(\left\{u_{i}\right\}_{i=1}^{n}\right)$ where $u_{i} \in \mathbb{R}^{r}$. Then $E \in \operatorname{ext}\left(\mathcal{E}_{n}\right)$ if and only if

$$
\operatorname{span}\left(u_{1} u_{1}^{\top}, \ldots, u_{n} u_{n}^{\top}\right)=\mathcal{S}_{r} .
$$

Equivalently, we have that $E \in \operatorname{ext}\left(\mathcal{E}_{n}\right)$ if and only if

$$
\operatorname{rank}(E \circ E)=\left(\begin{array}{c}
\operatorname{rank}(E)+1 \\
2
\end{array}\right)
$$

where $X \circ Y$ denotes the entrywise product of $X$ and $Y$.

Combining Lemma 2.1 with Theorem 2.2 we have the following useful result.

Theorem 2.3. Consider $E \in \operatorname{ext}\left(\mathcal{E}_{n}\right)$ with $r=\operatorname{rank}(E)$ and let $E=\operatorname{Gram}\left(\left\{u_{i}\right\}_{i=1}^{n}\right)$ where $\operatorname{span}\left(\left\{u_{i}\right\}_{i=1}^{n}\right)=\mathbb{R}^{r}$. For any two operator valued quadratic maps $Q_{1}, Q_{2}: \mathbb{R}^{r} \rightarrow \mathcal{H}_{d}$ (for some $d \geq 1$ ) satisfying $Q_{1}\left(u_{i}\right)=Q_{2}\left(u_{i}\right)$, for all $i \in[n]$ we have that $Q_{1}=Q_{2}$.

Proof. Consider the operator valued quadratic form $Q:=Q_{1}-Q_{2}$. By assumption we have that $Q\left(u_{i}\right)=0$ for all $i \in[n]$. As $E \in \operatorname{ext}\left(\mathcal{E}_{n}\right)$, by Theorem 2.2 we have that (14) holds. Lastly, Lemma 2.1 implies that $Q=0$.

\subsection{Bipartite correlation matrices}

By definition of $\pi$ (recall (4)) and $\mathcal{E}_{n+m}$ we have:

$$
\pi\left(\mathcal{E}_{n+m}\right)=\left\{C \in[-1,1]^{n \times m}: c_{i j}=\left\langle u_{i}, v_{j}\right\rangle \text {, where }\left\|u_{i}\right\|=\left\|v_{j}\right\|=1, \forall i, j\right\} .
$$

For a bipartite correlation matrix $C \in \pi\left(\mathcal{E}_{n+m}\right)$, any $E \in \mathcal{E}_{n+m}$ with $\pi(X)=C$ is called a completion of $C$. As was shown in [23], all equality constraints in (16) can be relaxed with inequalities, without enlarging the set. For completeness we give a proof in the Appendix.

Lemma 2.4 ([23]). For all $n, m \geq 1$ we have that

$$
\pi\left(\mathcal{E}_{n+m}\right)=\left\{C \in[-1,1]^{n \times m}: c_{i j}=\left\langle u_{i}, v_{j}\right\rangle \text {, where }\left\|u_{i}\right\|,\left\|v_{i}\right\| \leq 1, \forall i, j\right\} .
$$

Given a bipartite correlation $C \in \pi\left(\mathcal{E}_{n+m}\right)$, any family of vectors $\left\{u_{i}\right\}_{i=1}^{n},\left\{v_{j}\right\}_{j=1}^{m}$ satisfying $c_{i j}=\left\langle u_{i}, v_{j}\right\rangle$ and $\left\|u_{i}\right\|,\left\|v_{j}\right\| \leq 1$, for all $i \in[n], j \in[m]$ is called a $C$-system.

In the next result we summarize some basic properties of the set of completions of extreme bipartite correlations. For completeness, we give a short proof in the Appendix.

Lemma $2.5([23])$. For any $C=\left(c_{i j}\right) \in \operatorname{ext}\left(\pi\left(\mathcal{E}_{n+m}\right)\right)$ we have that:

(i) All C-systems necessarily consist of unit vectors;

(ii) For any $C$-system $\left\{u_{i}\right\}_{i=1}^{n},\left\{v_{j}\right\}_{j=1}^{m}$ we have that $\operatorname{span}\left(\left\{u_{i}\right\}_{i=1}^{n}\right)=\operatorname{span}\left(\left\{v_{j}\right\}_{j=1}^{m}\right)$;

(iii) There exists a unique matrix $E_{C} \in \mathcal{E}_{n+m}$ satisfying $\pi\left(E_{C}\right)=C$. Furthermore, we have $E_{C}=\left(\begin{array}{cc}A & C \\ C^{\top} & B\end{array}\right) \in \operatorname{ext}\left(\mathcal{E}_{n+m}\right)$ and $\operatorname{rank}\left(E_{C}\right)=\operatorname{rank}(A)=\operatorname{rank}(B)=\operatorname{rank}(C)$. 


\subsection{Matrix factorizations of correlation matrices}

We are finally ready to show that any correlation matrix admits a matrix factorization as defined in Theorem 1.2.

Lemma 2.6. Consider a real symmetric matrix $E \in \mathcal{S}_{n+m}$. The following are equivalent:

(a) $E \in \mathcal{E}_{n+m}$, i.e., there exist real unit vectors $\left\{a_{i}\right\}_{i=1}^{n},\left\{b_{j}\right\}_{j=1}^{m}$ such that

$$
E=\operatorname{Gram}\left(a_{1}, \ldots, a_{n}, b_{1}, \ldots, b_{m}\right) .
$$

(b) There exist $d \times d$ Hermitian matrices $\left\{A_{i}\right\}_{i=1}^{n},\left\{B_{j}\right\}_{j=1}^{m}$ such that

(i) $E=\operatorname{Gram}\left(A_{1}, \ldots, A_{n}, B_{1}, \ldots, B_{m}\right)$;

(ii) $A_{i}^{2}=B_{j}^{2}=\frac{1}{d} I_{d}$, for all $i \in[n], j \in[m]$.

(c) There exist $d \times d$ Hermitian matrices $\left\{X_{i}\right\}_{i=1}^{n},\left\{Y_{j}\right\}_{j=1}^{m}, K$ such that

(i) $E=\operatorname{Gram}\left(K X_{1}, \ldots, K X_{n}, Y_{1} K, \ldots, Y_{m} K\right)$;

(ii) $X_{i}^{2}=Y_{j}^{2}=I_{d}$, for all $i \in[n], j \in[m]$;

(iii) $\operatorname{Tr}\left(K^{2}\right)=1$ and $K$ is positive definite.

Proof. $(a) \Longrightarrow(b)$. Let $d=2^{\lfloor r / 2\rfloor}$. By the properties of the map $\gamma_{r}$ (recall (11)) we have that $\left\langle a_{i}, b_{j}\right\rangle=\left\langle\frac{\gamma\left(a_{i}\right)}{\sqrt{d}}, \frac{\gamma\left(b_{j}\right)}{\sqrt{d}}\right\rangle$ and $\gamma\left(a_{i}\right)^{2}=\gamma\left(b_{j}\right)^{2}=I_{d}$, for all $i \in[n], j \in[m]$.

$(b) \Longrightarrow(c)$. Set $K=d^{-1 / 2} I_{d}$ and $X_{i}=\sqrt{d} A_{i}, Y_{j}=\sqrt{d} B_{j}$, for all $i \in[n], j \in[m]$.

$(c) \Longrightarrow(a)$. For all $i \in[n]$ let $\tilde{a}_{i}=\operatorname{vec}\left(K X_{i}\right)$ and set $a_{i}=\left(\operatorname{Re}\left(\tilde{a}_{i}\right), \operatorname{Im}\left(\tilde{a}_{i}\right)\right)$. For all $j \in[m]$ define $\tilde{b}_{j}$ and $b_{j}$ analogously. As the entries of $E$ are real numbers we have that $E=\operatorname{Gram}\left(a_{1}, \ldots, a_{n}, b_{1}, \ldots, b_{m}\right)$. Lastly, note that

$$
1=\operatorname{Tr}\left(K^{2}\right)=\operatorname{Tr}\left(X_{i}^{2} K^{2}\right)=\left\langle K X_{i}, K X_{i}\right\rangle=\left\|\tilde{a}_{i}\right\|^{2}=\left\|a_{i}\right\|^{2}, \text { for all } i \in[n] .
$$

Similarly we have that $\left\|b_{j}\right\|=1$, for all $j \in[m]$.

We refer to any family of matrices satisfying condition $(c)$ from Lemma 2.6 as a matrix factorization of $E$. As already described in the introduction, our goal is to show that for extreme points of $\mathcal{E}_{n+m}$, we can place a lower bound on the size of matrix factorizations, which is exponential in terms of $\operatorname{rank}(E)$. We note in passing that using the same arguments we can also lower bound matrix factorizations satisfying condition $(b)$ from Lemma 2.6. Nevertheless, lower bounds for matrix factorizations of type $(c)$ are stronger. 


\subsection{Proof of the main technical result}

In this section we prove Theorem 1.2. This will follow as a consequence of the following.

Lemma 2.7. Let $E=\left(\begin{array}{cc}A & C \\ C^{\top} & B\end{array}\right)$ be an extreme point of $\mathcal{E}_{n+m}$ where $\operatorname{rank}(A)=\operatorname{rank}(E)=n$. Consider a family of $d \times d$ Hermitian operators $\left\{X_{i}\right\}_{i=1}^{n}$ satisfying

$$
X_{i}^{2}=I_{d}, \quad \text { for all } i \in[n], \quad \text { and }\left(\sum_{i=1}^{n} \lambda_{i j} X_{i}\right)^{2}=I_{d}, \text { for all } j \in[m],
$$

where $\Lambda=\left(\lambda_{i j}\right)$ is an $n \times m$ matrix satisfying $C=A \Lambda$ and $B=\Lambda^{\top} A \Lambda$ (the fact that such a matrix exists follows from (5)). Then we have that

$$
\left(\sum_{i=1}^{n} \mu_{i} X_{i}\right)^{2}=\left(\mu^{\top} A \mu\right) I_{d}, \text { for all } \mu=\left(\mu_{i}\right) \in \mathbb{R}^{n} .
$$

In particular, the algebra $\mathbb{C}\left[X_{1}, \ldots, X_{n}\right]$ generated by $\left\{X_{i}\right\}_{i=1}^{n}$ is isomorphic to the rank-n Clifford algebra $\mathcal{C}_{n}$ and thus, the size of the matrices $X_{1}, \ldots, X_{n}$ is lower bounded by $2^{\lfloor n / 2\rfloor}$.

Proof. Consider vectors $\left\{a_{i}\right\}_{i=1}^{n},\left\{b_{j}\right\}_{j=1}^{m} \subseteq \mathbb{R}^{n}$ satisfying

$$
E=\operatorname{Gram}\left(a_{1}, \ldots, a_{n}, b_{1}, \ldots, b_{m}\right) \text { and } \operatorname{span}\left(\left\{a_{i}\right\}_{i=1}^{n}\right)=\mathbb{R}^{n} \text {. }
$$

Using (6) combined with the fact that $C=A \Lambda$ and $B=\Lambda^{\top} A \Lambda$ we get

$$
b_{j}=\sum_{i=1}^{n} \lambda_{i j} a_{i}, \text { for all } j \in[m] .
$$

For $i \in\{1,2\}$ we define operator valued quadratic maps $Q_{i}: \mathbb{R}^{n} \rightarrow \mathcal{H}_{d}$ as

$$
Q_{1}\left(\sum_{i=1}^{n} \mu_{i} a_{i}\right):=\left(\sum_{i=1}^{n} \mu_{i} X_{i}\right)^{2} \quad \text { and } \quad Q_{2}\left(\sum_{i=1}^{n} \mu_{i} a_{i}\right):=\left\|\sum_{i=1}^{n} \mu_{i} a_{i}\right\|^{2} I_{d} .
$$

As the vectors $\left\{a_{i}\right\}_{i=1}^{n}$ form a basis of $\mathbb{R}^{n}$ the maps $Q_{1}$ and $Q_{2}$ are well-defined. Note that the claim (19) is equivalent to $Q_{1}=Q_{2}$. Thus, by Theorem 2.3 it suffices to show that

$$
Q_{1}\left(a_{i}\right)=Q_{2}\left(a_{i}\right), \forall i \in[n] \text { and } Q_{1}\left(b_{j}\right)=Q_{2}\left(b_{j}\right), \forall j \in[m] .
$$

First, note that

$$
Q_{1}\left(a_{i}\right)=X_{i}^{2}=I_{d}=Q_{2}\left(a_{i}\right) \text {, for all } i \in[n],
$$

where we use that $\left\|a_{i}\right\|=1$, for all $i \in[n]$. Furthermore, for all $j \in[m]$ we have

$$
Q_{1}\left(b_{j}\right)=Q_{1}\left(\sum_{i=1}^{n} \lambda_{i j} a_{i}\right)=\left(\sum_{i=1}^{n} \lambda_{i j} X_{i}\right)^{2}=I_{d}
$$

where for the first equality we use (20) and for the last equality (18). Similarly,

$$
Q_{2}\left(b_{j}\right)=Q_{2}\left(\sum_{i=1}^{n} \lambda_{i j} a_{i}\right)=\left\|\sum_{i=1}^{n} \lambda_{i j} a_{i}\right\|^{2} I_{d}=\left\|b_{j}\right\|^{2} I_{d}=I_{d}
$$


where we use that $\left\|b_{j}\right\|=1$, for all $j \in[m]$. Thus, (21) holds which in turn implies (19).

Lastly, as an immediate consequence of (19) we get that

$$
X_{i} X_{j}+X_{j} X_{i}=2 A_{i j} I_{d}, \text { for all } i, j \in[n] .
$$

Let $A=\sum_{k=1}^{n} \lambda_{k} u_{k} u_{k}^{\top}$ be a spectral decomposition of $A$. By assumption $A$ is positive definite and thus, $\lambda_{k}>0$ for all $k \in[n]$. Setting

$$
X_{k}^{\prime}:=\lambda_{k}^{-1 / 2} \sum_{i=1}^{n} u_{k}(i) X_{i}, \text { for all } k \in[n],
$$

we have that

$$
X_{i}^{\prime} X_{j}^{\prime}+X_{j}^{\prime} X_{i}^{\prime}=2 \delta_{i, j} I_{d}, \text { for all } i, j \in[n],
$$

and thus $\mathbb{C}\left[X_{1}, \ldots, X_{n}\right]$ is isomorphic to the rank- $n$ Clifford algebra $\mathcal{C}_{n}$.

We now give the proof of Theorem 1.2. We restate it below for the ease of the reader.

Theorem 2.8. Let $E=\left(\begin{array}{cc}A & C \\ C^{\top} & B\end{array}\right)$ be an extreme point of $\mathcal{E}_{n+m}$ where $\operatorname{rank}(A)=\operatorname{rank}(E)=n$. Consider $d \times d$ Hermitian matrices $\left\{X_{i}\right\}_{i=1}^{n},\left\{Y_{j}\right\}_{j=1}^{m}, K$ satisfying

(i) $E=\operatorname{Gram}\left(K X_{1}, \ldots, K X_{n}, Y_{1} K, \ldots, Y_{m} K\right)$;

(ii) $X_{i}^{2}=Y_{j}^{2}=I_{d}$, for all $i \in[n], j \in[m]$;

(iii) $\operatorname{Tr}\left(K^{2}\right)=1$ and $K$ is positive definite.

Then, the algebra $\mathbb{C}\left[X_{1}, \ldots, X_{n}\right]$ is isomorphic to the rank-n Clifford algebra $\mathcal{C}_{n}$. In particular, the size of the matrices $X_{1}, \ldots, X_{n}$ is lower bounded by $2^{\lfloor n / 2\rfloor}$.

Proof. As $\operatorname{rank}(E)=\operatorname{rank}(A)$, there exists an $n \times m$ matrix $\Lambda=\left(\lambda_{i j}\right)$ such that $C=A \Lambda$ and $B=\Lambda^{\top} A \Lambda$. Since $E=\operatorname{Gram}\left(K X_{1}, \ldots, K X_{n}, Y_{1} K, \ldots, Y_{m} K\right)$ it follows by (6) that

$$
Y_{j} K=\sum_{i=1}^{n} \lambda_{i j} K X_{i}, \text { for all } j \in[m],
$$

and as $K$ is positive definite (and hence invertible) we obtain

$$
Y_{j}=\sum_{i=1}^{n} \lambda_{i j} K X_{i} K^{-1}, \text { for all } j \in[m] .
$$

Lastly, define

$$
\tilde{X}_{i}=K X_{i} K^{-1} \text {, for all } i \in[n] .
$$

By assumption we have that $X_{i}^{2}=I_{d}$, for all $i \in[n]$, which implies that $\tilde{X}_{i}^{2}=I_{d}$. Furthermore, as $Y_{j}^{2}=I_{d}$ for all $j \in[m]$, it follows by (23) that

$$
\left(\sum_{i=1}^{n} \lambda_{i j} \tilde{X}_{i}\right)^{2}=I_{d}, \quad \text { for all } j \in[m] .
$$

The proof of the theorem is is concluded using Lemma 2.7. 


\section{Cpsd matrices with sub-exponential cpsd-rank}

In this section we use Theorem 1.2 to prove Theorem 1.1. The crux of the proof lies in the following result.

Theorem 3.1. For any $C=\left(c_{i j}\right) \in \operatorname{ext}\left(\mathcal{E}_{n}\right)$ the matrix

$$
P_{C}=\sum_{i, j=1}^{n} \frac{1}{4}\left(\begin{array}{ll}
1+c_{i j} & 1-c_{i j} \\
1-c_{i j} & 1+c_{i j}
\end{array}\right) \otimes e_{i} e_{j}^{\top},
$$

is cpsd and furthermore, cpsd-rank $\left(P_{C}\right) \geq 2^{\lfloor\operatorname{rank}(C) / 2\rfloor}$.

Proof. Let $C=\operatorname{Gram}\left(\left\{u_{i}\right\}_{i=1}^{n}\right)$ where $\left\{u_{i}\right\}_{i=1}^{n} \subseteq \mathbb{R}^{r}$ and $\left\|u_{i}\right\|=1, \forall i \in[n]$. As suggested by (25) we think of $P_{C}$ as an $n \times n$ block matrix where each block has size $2 \times 2$ and is indexed by $\{ \pm 1\}$.

We first show that $P_{C} \in \mathcal{C S}_{+}^{2 n}$. For this, set $d=2^{\lfloor r / 2\rfloor}$ and define

$$
\Gamma_{a}^{i}=\frac{I+a \gamma_{r}\left(u_{i}\right)}{2 \sqrt{d}}, \quad \text { for all } i \in[n], a \in\{ \pm 1\}
$$

and note that by the properties of the $\gamma_{r}$ map (recall (11)), these matrices are Hermitian psd. Furthermore, by direct calculation for all $i, j \in[n]$ and $a, b \in\{ \pm 1\}$ we have that

$$
\left\langle\Gamma_{a}^{i}, \Gamma_{b}^{j}\right\rangle=\frac{d+a b\left\langle\gamma_{r}\left(u_{i}\right), \gamma_{r}\left(u_{j}\right)\right\rangle}{4 d}=\frac{1+a b\left\langle u_{i}, u_{j}\right\rangle}{4}=\frac{1+a b c_{i j}}{4},
$$

which shows that the matrices $\left\{\Gamma_{a}^{i}: i \in[n], a \in\{ \pm 1\}\right\}$ form a cpsd-factorization for $P_{C}$. Next we proceed to show the lower bound.

Let $\left\{P_{a}^{i}: i \in[n], a \in\{ \pm 1\}\right\}$ be a size-optimal cpsd-factorization for $P_{C}$. We now identify some useful properties of these matrices which we use later in the proof. As the entries of $P_{C}$ in each $2 \times 2$ block sum up to one we get

$$
\sum_{a \in\{ \pm 1\}} P_{a}^{i}=\sum_{a \in\{ \pm 1\}} P_{a}^{j}, \quad \text { for all } i, j \in[n] .
$$

For all $i \in[n]$ set

$$
K:=\sum_{a \in\{ \pm 1\}} P_{a}^{i}
$$

which is well-defined by (28). Furthermore, note that $K$ is psd and $\langle K, K\rangle=1$.

Since the cpsd factorization is size-optimal we may assume without loss of generality that $K$ is diagonal and positive definite. Indeed, let $K=Q \Lambda Q^{*}$ be its spectral decomposition. Clearly, the matrices $\left\{Q^{*} P_{a}^{i} Q: i \in[n], a \in\{ \pm 1\}\right\}$ are Hermitian positive semidefinite and as $Q$ is unitary, it follows that they form a cpsd-factorization for $P_{C}$. As a consequence, if $K$ was rank-deficient, by restricting the matrices $\left\{Q^{*} P_{a}^{i} Q: i \in[n], a \in\{ \pm 1\}\right\}$ onto the support of $K$, we would get another cpsd-factorization of smaller size. This contradicts the assumption that $\left\{P_{a}^{i}: i \in[n], a \in\{ \pm 1\}\right\}$ was size-optimal. 
Our next goal is to use the cpsd-factorization $\left\{P_{a}^{i}: i \in[n], a \in\{ \pm 1\}\right\}$ to obtain the matrix factorization to which Theorem 1.2 will be applied. As $K$ invertible we have that

$$
\left\langle P_{a}^{i}, P_{b}^{j}\right\rangle=\left\langle K\left(K^{-1 / 2} P_{a}^{i} K^{-1 / 2}\right),\left(K^{-1 / 2} P_{b}^{j} K^{-1 / 2}\right) K\right\rangle=\left\langle K \tilde{P}_{a}^{i}, \tilde{P}_{b}^{j} K\right\rangle, \quad \forall i, j \in[n],
$$

where we define

$$
\tilde{P}_{a}^{i}:=K^{-1 / 2} P_{a}^{i} K^{-1 / 2}, \quad \text { for all } i \in[n], a \in\{ \pm 1\} .
$$

An easy calculation shows that

$$
c_{i j}=\sum_{a, b \in\{ \pm 1\}} a b\left(\frac{1+a b c_{i j}}{4}\right)=\sum_{a, b \in\{ \pm 1\}} a b\left\langle P_{a}^{i}, P_{b}^{j}\right\rangle=\sum_{a, b \in\{ \pm 1\}} a b\left\langle K \tilde{P}_{a}^{i}, \tilde{P}_{b}^{j} K\right\rangle,
$$

where for the second equality we use that $\left\{P_{a}^{i}: i \in[n], a \in\{ \pm 1\}\right\}$ is a cpsd-factorization for $P_{C}$ and the third equality follows from (30). Setting

$$
X_{i}:=\tilde{P}_{1}^{i}-\tilde{P}_{-1}^{i}, \quad \forall i \in[n],
$$

it follows by (32) that

$$
c_{i j}=\left\langle K X_{i}, X_{j} K\right\rangle, \quad \forall i, j \in[n] .
$$

By (29) we have $\sum_{a \in\{ \pm 1\}} P_{a}^{i}=K$ which implies that $\tilde{P}_{1}^{i}+\tilde{P}_{-1}^{i}=I$, for all $i \in[n]$. Thus, for any $i \in[n]$, the Hermitian matrix $X_{i}=\tilde{P}_{1}^{i}-\tilde{P}_{-1}^{i}=2 \tilde{P}_{1}^{i}-I$ has spectrum in $[-1,1]$, i.e., $X_{i}^{2} \preceq I$. In fact, as $C \in \operatorname{ext}\left(\mathcal{E}_{n}\right)$, it follows by Lemma $2.5(i)$ that

$$
X_{i}^{2}=I, \quad \text { for all } i \in[n] .
$$

Note that the same argument was given in the proof of Theorem 1.3.

We are now ready to conclude the proof. As $C \in \operatorname{ext}\left(\mathcal{E}_{n}\right)$, Theorem 2.5 (iii) implies that $C$ has a unique elliptope completion $E_{C} \in \mathcal{E}_{2 n}$, which moreover is an extreme point of $\mathcal{E}_{2 n}$. Nevertheless, as $C \in \mathcal{E}_{n}$, the matrix $(\underset{C}{C} \underset{C}{C})$ is clearly an elliptope completion of $C$. As a consequence we have that

$$
E_{C}=\left(\begin{array}{ll}
C & C \\
C & C
\end{array}\right) \in \operatorname{ext}\left(\mathcal{E}_{2 n}\right)
$$

which is the matrix to which we will apply Theorem 1.2. The last step is to exhibit a matrix factorization for $E_{C}$. For this consider the psd matrix

$$
E_{C}^{\prime}:=\operatorname{Gram}\left(K X_{1}, \ldots, K X_{n}, X_{1} K, \ldots, X_{n} K\right) .
$$

By (34) we have

$$
\left\langle K X_{i}, K X_{i}\right\rangle=\operatorname{Tr}\left(X_{i}^{2} K^{2}\right)=\operatorname{Tr}\left(K^{2}\right)=1, \quad \forall i \in[n],
$$

and thus, $E_{C}^{\prime}$ is an element of the elliptope $\mathcal{E}_{2 n}$. Finally, by (33) it follows that $E_{C}^{\prime}$ is an elliptope completion of $C$. Thus, again by Lemma $2.5($ iii $)$ we get that $E_{C}=E_{C}^{\prime}$, i.e.,

$$
\left(\begin{array}{ll}
C & C \\
C & C
\end{array}\right)=\operatorname{Gram}\left(K X_{1}, \ldots, K X_{n}, X_{1} K, \ldots, X_{n} K\right) \in \operatorname{ext}\left(\mathcal{E}_{2 n}\right),
$$

and the proof is concluded by Theorem 1.2.

To prove Theorem 1.1, it remains to combine Theorem 3.1 with the following wellknown fact: For any $X \in \operatorname{ext}\left(\mathcal{E}_{n}\right)$ we have that $\operatorname{rank}(X) \leq r_{\max }(n)$. Furthermore, for any integer $r \in\left[1, r_{\max }(n)\right]$ there exists a matrix $X_{r} \in \operatorname{ext}\left(\mathcal{E}_{n}\right)$ with $r=\operatorname{rank}\left(X_{r}\right)$ [13]. 


\section{Relation to Tsirelson's work}

In this section we explain the connection between quantum information theory and bipartite correlation matrices. The set $\pi\left(\mathcal{E}_{n+m}\right)$ was studied by Tsirelson due to its relevance to quantum information theory. Algebraically, this is captured by the following result found in [23, Theorem 2.1]. We give a brief proof for completeness.

Theorem 4.1. Let $C=\left(c_{i j}\right) \in[-1,1]^{n \times m}$. Then, $C \in \pi\left(\mathcal{E}_{n+m}\right)$ if and only if there exist Hermitian matrices $\left\{M_{i}\right\}_{i=1}^{n},\left\{N_{j}\right\}_{j=1}^{m} \subseteq \mathcal{H}_{d}$ and a Hermitian matrix $\rho \in \mathcal{H}_{d^{2}}$ such that

(i) $M_{i}^{2} \preceq I, N_{j}^{2} \preceq I$, for all $i \in[n], j \in[m]$;

(ii) $\rho$ is positive semidefinite with $\operatorname{Tr}(\rho)=1$;

(iii) $c_{i j}=\operatorname{Tr}\left(\left(M_{i} \otimes N_{j}\right) \rho\right)$, for all $i \in[n], j \in[m]$.

Proof. Let $C \in \pi\left(\mathcal{E}_{n+m}\right)$ and consider vectors $\left\{u_{i}\right\}_{i=1}^{n},\left\{v_{j}\right\}_{j=1}^{m} \subseteq \mathbb{R}^{r}$ satisfying $c_{i j}=$ $\left\langle u_{i}, v_{j}\right\rangle$ and $\left\|u_{i}\right\|,\left\|v_{j}\right\| \leq 1$, for all $i \in[n], j \in[m]$ (such vectors exist by (17)). Then,

$$
c_{i j}=\left\langle u_{i}, v_{j}\right\rangle=\frac{\operatorname{Tr}\left(\gamma_{r}\left(u_{i}\right) \gamma_{r}\left(v_{j}\right)\right)}{d}=\operatorname{Tr}\left(\left(\gamma_{r}\left(u_{i}\right) \otimes \gamma_{r}\left(v_{i}\right)^{\top}\right) \psi_{d} \psi_{d}^{*}\right), \forall i \in[n], j \in[m],
$$

where $d=2^{\lfloor r / 2\rfloor}$ and $\psi_{d}:=d^{-1 / 2} \sum_{i=1}^{d} e_{i} \otimes e_{i} \in \mathbb{C}^{d} \otimes \mathbb{C}^{d}$. To prove (36), for the second equality we use (11) and for the third one that $\psi_{d}^{*}(A \otimes B) \psi_{d}=\frac{1}{d} \operatorname{Tr}\left(A B^{\top}\right), \forall A, B \in \mathcal{M}_{d}$.

Conversely, consider matrices $\left\{M_{i}\right\}_{i=1}^{n},\left\{N_{j}\right\}_{j=1}^{m}$ and $\rho$ satisfying $(i),(i i)$ and (iii). Setting $A_{i}=M_{i} \otimes I$ and $B_{j}=I \otimes N_{j}$ for all $i \in[n], j \in[m]$ we get that

$$
c_{i j}=\operatorname{Tr}\left(A_{i} B_{j} \rho\right)=\left\langle\rho^{1 / 2} A_{i}, \rho^{1 / 2} B_{j}\right\rangle, \forall i \in[n], j \in[m] .
$$

For all $i \in[n]$ let $\tilde{u}_{i}=\operatorname{vec}\left(\rho^{1 / 2} A_{i}\right)$ and $u_{i}=\left(\operatorname{Re}\left(\tilde{u}_{i}\right), \operatorname{Im}\left(\tilde{u}_{i}\right)\right)$. For all $j \in[m]$ define $\tilde{v}_{j}$ and $v_{j}$ analogously. As the entries of $C$ are real numbers we get that $c_{i j}=\left\langle u_{i}, v_{j}\right\rangle$, for all $i \in[n], j \in[m]$. Lastly, note that

$$
\left\|u_{i}\right\|^{2}=\left\|\tilde{u}_{i}\right\|^{2}=\operatorname{Tr}\left(A_{i}^{2} \rho\right) \leq \operatorname{Tr}(\rho)=1,
$$

where for the inequality we use that $A_{i}^{2} \preceq I$. Similarly, we get $\left\|v_{j}\right\| \leq 1, \forall j \in[m]$.

The algebraic representation of the set of bipartite correlations given above turns out to have operational interpretation within the context of quantum information theory.

Definition 4.2. A matrix $C=\left(c_{i j}\right) \in[-1,1]^{n \times m}$ is called a quantum correlation matrix if there exist Hermitian matrices $\left\{M_{i}\right\}_{i=1}^{n},\left\{N_{j}\right\}_{j=1}^{m} \subseteq \mathcal{H}_{d}$ and a Hermitian matrix $\rho \in \mathcal{H}_{d^{2}}$ (for some $d \geq 1$ ) satisfying conditions $(i),(i i)$ and (iii) from Theorem 4.1.

We refer to any such family of matrices as a tensor product representation of $C$ with local dimension $d$. In this section we use Theorem 1.2 to lower bound the local dimension of tensor product representations corresponding to extreme points of the set of quantum correlations.

As a first step we show that without loss of generality, we may only consider tensor product representations where $\rho$ is a rank-one matrix. This is known but we give a short proof for completeness. 
Lemma 4.3 ([21]). For any $C \in \operatorname{ext}\left(\pi\left(\mathcal{E}_{n+m}\right)\right)$, the minimum local dimension of a tensor product representation can be achieved by a rank-one representation $\rho=\psi \psi^{*}$ satisfying:

$$
\psi=\sum_{i=1}^{d} \lambda_{i} e_{i} \otimes e_{i}^{*} \in \mathbb{C}^{d} \otimes \mathbb{C}^{d}, \quad \lambda_{i}>0(\forall i \in[d]), \quad \text { and } \quad \sum_{i=1}^{d} \lambda_{i}^{2}=1 .
$$

Proof. The extreme points of the compact convex set $\{\rho: \rho \succeq 0, \operatorname{Tr}(\rho)=1\}$ are matrices of the form $\phi \phi^{*}$, where $\|\phi\|=1$. Thus, by the extremality assumption, for every tensor product representation of $C$ we have that $\rho=\phi \phi^{*}$, for some vector $\phi$ with $\|\phi\|=1$.

It remains to show that given a rank-one tensor product representation of $C$ with local dimension $d^{\prime}$, i.e.,

$$
c_{i j}=\phi^{*}\left(M_{i} \otimes N_{j}\right) \phi, \forall i, j \text { where } \phi \in \mathbb{C}^{d^{\prime}} \otimes \mathbb{C}^{d^{\prime}},
$$

we can construct another rank-one tensor product representation of $C$ satisfying (37), whose local dimension is upper bounded by $d^{\prime}$.

For this, consider a Schmidt decomposition of $\phi$, i.e., $\phi=\sum_{k=1}^{d} \lambda_{k} x_{k} \otimes y_{k}$, where $\left\{\lambda_{k}\right\}_{k=1}^{d}$ are strictly positive, $\sum_{k=1}^{d} \lambda_{k}^{2}=1$, and $\left\{y_{k}\right\}_{k=1}^{d},\left\{x_{k}\right\}_{k=1}^{d} \subseteq \mathbb{C}^{d^{\prime}}$ are orthonormal vectors. Clearly we have that $d \leq d^{\prime}$. Define the $d \times d^{\prime}$ matrices $U:=\sum_{k=1}^{d} e_{k} x_{k}^{*}$ and $V:=\sum_{k=1}^{d} e_{k} y_{k}^{*}$, and note that the vector

$$
\psi:=(U \otimes V) \phi=\sum_{k=1}^{d} \lambda_{k} e_{k} \otimes e_{k} \in \mathbb{C}^{d} \otimes \mathbb{C}^{d},
$$

satisfies $\operatorname{Tr}\left(\psi \psi^{*}\right)=1$. Moreover, as $U U^{*}=V V^{*}=I_{d}$, it follows that the Hermitian $d \times d$ matrices $\left\{\tilde{M}_{i}:=U M_{i} U^{*}\right\}_{i=1}^{n}$ and $\left\{\tilde{N}_{j}:=V N_{j} V^{*}\right\}_{j=1}^{m}$ have spectrum in $[-1,1]$. To see this, recall that for any Hermitian matrix $X$, the condition $X^{2} \preceq I$ is equivalent to $\left(\begin{array}{cc}I & X \\ X & I\end{array}\right) \succeq 0$. (e.g. by using Schur complements). By assumption we have that $M_{i}^{2} \preceq I$ and $N_{j}^{2} \preceq I$, for all $i, j$. Thus, we have that $\left(\begin{array}{cc}I_{d^{\prime}} & M_{i} \\ M_{i} & I_{d^{\prime}}\end{array}\right) \succeq 0$ which implies

$$
\left(\begin{array}{cc}
I_{d} & \tilde{M}_{i} \\
\tilde{M}_{i} & I_{d}
\end{array}\right)=\left(\begin{array}{cc}
U & 0 \\
0 & U
\end{array}\right)\left(\begin{array}{cc}
I_{d^{\prime}} & M_{i} \\
M_{i} & I_{d^{\prime}}
\end{array}\right)\left(\begin{array}{cc}
U^{*} & 0 \\
0 & U^{*}
\end{array}\right) \succeq 0
$$

Similarly, we get $\tilde{N}_{j}^{2} \preceq I, \forall j \in[m]$. Lastly, an easy calculation gives that

$$
c_{i j}=\phi^{*}\left(M_{i} \otimes N_{j}\right) \phi=\psi^{*}\left(\tilde{M}_{i} \otimes \tilde{N}_{j}\right) \psi, \quad \forall i \in[n], j \in[m],
$$

and the proof is concluded.

As an application application of Theorem 1.2 we now prove Theorem 1.3.

Proof. (of Theorem 1.3) By Lemma 4.3 we may only consider rank-one tensor product representations, i.e., $c_{i j}=\psi^{*}\left(M_{i} \otimes N_{j}\right) \psi$, where $M_{i}^{2} \preceq I, N_{j}^{2} \preceq I$, for all $i \in[n], j \in[m]$ and $\psi$ has the form given in (37). Set $K:=\operatorname{vec}(\psi)=\sum_{i=1}^{d} \lambda_{i} e_{i} e_{i}^{*}$ and note that $K$ is positive definite (and even diagonal) and satisfies $\operatorname{Tr}\left(K^{2}\right)=1$. By (7) we have that

$$
c_{i j}=\operatorname{vec}(K)^{*}\left(M_{i} \otimes N_{j}\right) \operatorname{vec}(K)=\operatorname{Tr}\left(K M_{i} K N_{j}^{\boldsymbol{\top}}\right)=\left\langle K X_{i}, Y_{j} K\right\rangle,
$$


where $X_{i}:=M_{i}$ and $Y_{j}:=N_{j}^{\top}$. Note that since $N_{j}$ is Hermitian the same holds for $Y_{j}$.

Clearly $X_{i}^{2} \preceq I$, and since a matrix and its transpose have the same eigenvalues we also have that $Y_{j}^{2} \preceq I$. We now show that in fact $X_{i}^{2}=Y_{j}^{2}=I$, for all $i \in[n], j \in[m]$. Towards a contradiction, assume there exists $i^{*} \in[n]$ such that $X_{i^{*}}^{2} \prec I$. Then,

$$
\left\langle K X_{i^{*}}, K X_{i^{*}}\right\rangle=\operatorname{Tr}\left(X_{i^{*}}^{2} K^{2}\right)<\operatorname{Tr}\left(K^{2}\right)=1 .
$$

Thus, by vectorizing the matrices $\left\{K X_{i}\right\}_{i=1}^{n}$ and $\left\{Y_{j} K\right\}_{j=1}^{m}$, in view of (38) we get a $C$-system where one of the vectors has norm strictly less than one. Nevertheless, as $C \in \operatorname{ext}\left(\pi\left(\mathcal{E}_{n+m}\right)\right)$, this possibility has been already excluded in Lemma $2.5(i)$.

Since $X_{i}^{2}=Y_{j}^{2}=I$, for all $i \in[n], j \in[m]$, the matrix $\operatorname{Gram}\left(\left\{K X_{i}\right\}_{i},\left\{Y_{j} K\right\}_{j}\right)$ is an elliptope completion of $C$. On the other hand, since $C \in \operatorname{ext}\left(\pi\left(\mathcal{E}_{n+m}\right)\right)$, we have seen in Lemma 2.5 (iii) that $C$ has a unique completion $E_{C}=\left(\underset{C^{\top}}{A}{ }_{B}^{C}\right)$ where $\operatorname{rank}\left(E_{C}\right)=$ $\operatorname{rank}(A)=\operatorname{rank}(B)=\operatorname{rank}(C)$ and $E_{C} \in \operatorname{ext}\left(\mathcal{E}_{n+m}\right)$. Consequently, we have that

$$
E_{C}=\operatorname{Gram}\left(\left\{K X_{i}\right\}_{i},\left\{Y_{j} K\right\}_{j}\right),
$$

and the claim follows by applying Theorem 1.2.

We note that Theorem 1.3 essentially follows from Tsirelson's seminal work [23], although it is not explicitly stated there (it is mentioned in [24] albeit without proof). Indeed, in [23] Tsirelson studies the properties of another family of matrix representations of quantum correlations called commuting representations, in the case where the ambient Hilbert space is finite-dimensional or countably infinite. His main result is that for any $C \in \operatorname{ext}\left(\pi\left(\mathcal{E}_{n+m}\right)\right)$, the matrices in a (nondegenerate) commuting representation correspond to a representation of an appropriate Clifford algebra [23, Theorem 3.1]. As a consequence, the dimension of any commuting representation of $C \in \operatorname{ext}\left(\pi\left(\mathcal{E}_{n+m}\right)\right)$ is lower bounded by $4^{\lfloor\operatorname{rank}(C) / 2\rfloor}$ (for a concise proof of this fact see [12, Theorem 4.4] or [19, Theorem 25]). On the other hand, it is well-known and easy to see that any tensor product representation with local dimension $d$ gives rise to a commuting representation of size $d^{2}$. Putting everything together we arrive at Theorem 1.3.

Interestingly, Theorem 1.2 generalizes Theorem 1.3 since there exist matrices $\left(\begin{array}{cc}C_{C^{\top}} & C \\ B\end{array}\right) \in$ $\operatorname{ext}\left(\mathcal{E}_{n+m}\right)$ for which $C \notin \operatorname{ext}\left(\pi\left(\mathcal{E}_{n+m}\right)\right)$. To give a concrete example define

$$
u_{i i}=e_{i} \quad(1 \leq i \leq r), \quad \text { and } \quad u_{i j}=\frac{e_{i}+e_{j}}{\sqrt{2}} \quad(1 \leq i<j \leq r)
$$

and let $E$ be the Gram matrix of the $u_{i j}(1 \leq i \leq j \leq r)$ ordered lexicographically. Using (15) one can easily verify that $E$ is an extreme point of the $\left(\begin{array}{c}r+1 \\ 2\end{array}\right)$-dimensional elliptope. On the other hand, let $C$ be the submatrix of $E$ obtained by restricting to the first $r$ rows and the columns indexed by pairs $(i, j)$ in the range $2 \leq i \leq j \leq r$. Clearly, $C$ is a $r \times\left(\begin{array}{l}r \\ 2\end{array}\right)$ bipartite correlation matrix but it is an not extreme point of $\pi\left(\mathcal{E}_{r+\left(\begin{array}{l}r \\ 2\end{array}\right)}\right)$. This is an immediate consequence of Lemma $2.5(i i)$, since $e_{1} \notin \operatorname{span}\left(u_{i j}: 2 \leq i \leq j \leq r\right)$.

\section{References}

[1] D. Avis, S. Moriyama, and M. Owari. From Bell inequalities to Tsirelson's theorem: A survey. IEICE Trans. on Fundamentals, E92-A(5):1254-1267, 2009. 
[2] H. Barnum, J. Barrett, M. Leifer, and A. Wilce. A general no-cloning theorem. Phys. Rev. Lett., 99:240501, 2007.

[3] A. Berman and N. Shaked-Monderer. Completely Positive Matrices. World Scientific, 2003.

[4] I. N. Bomze, W. Schachinger, and R. Ullrich. New lower bounds and asymptotics for the cp-rank. SIAM. J. Matrix Anal. A., 36(1):20-37, 2015.

[5] S. Burer. On the copositive representation of binary and continuous nonconvex quadratic programs. Math. Program. Ser. A, 120:479-495, 2009.

[6] M. Deza and M. Laurent. Geometry of cuts and metrices. Springer, 1997.

[7] J. Drew, C. Johnson, and R. Loewy. Completely positive matrices associated with M-matrices. Linear Multilinear A., 37(4):303-310, 1994.

[8] K. Dykema, V. I. Paulsen, and J. Prakash. Non-closure of the set of quantum correlations via graphs. arXiv:1709.05032.

[9] H. Fawzi, J. Gouveia, P. Parrilo, R. Z. Robinson, and R. Thomas. Positive semidefinite rank. Math. Program., 153(1):133-177, 2015.

[10] R. Goodman and N. R. Wallach. Symmetry, Representations, and Invariants. Springer, 2009.

[11] M. S. Gowda, R. Sznajder, and J. Tao. The automorphism group of a completely positive cone and its Lie algebra. Linear Alg. Appl., 438:3862-3871, 2013.

[12] S. Gribling, D. de Laat, and M. Laurent. Matrices with high completely positive semidefinite rank. Linear Alg. Appl., 513:122-148, May 2017.

[13] R. Grone, S. Pierce, and W. Watkins. Extremal correlation matrices. Linear Alg. Appl., 132(537):63-70, 1990.

[14] M. Laurent and T. Piovesan. Conic approach to quantum graph parameters using linear optimization over the completely positive semidefinite cone. SIAM J. Optimiz., 25(4):2461-2493, 2015.

[15] C.-K. Li and B.-S. Tam. A note on extremal correlation matrices. SIAM. J. Matrix Anal. A., 15(536):903-908, 1994.

[16] M. Musat and M. Rørdam. Non-closure of quantum correlation matrices and factorizable channels that require infinite dimensional ancilla. arXiv:1806.10242.

[17] T. Netzer and A. Thom. Polynomials with and without determinantal representations. Linear Alg. Appl., 437(7):1579-1595, 2012.

[18] T. Netzer and A. Thom. Hyperbolic polynomials and generalized clifford algebras. Discrete Comput. Geom., 51(4):802-814, 2014. 
[19] A. Prakash, J. Sikora, A. Varvitsiotis, and Z. Wei. Completely positive semidefinite rank. Math. Prog. Ser. A, 171(1-2):397-431, 2018.

[20] D. E. Roberson. Conic formulations of graph homomorphisms. J. Algebr. Comb., pages $1-37,2016$.

[21] J. Sikora and A. Varvitsiotis. Linear conic formulations for two-party correlations and values of nonlocal games. Math. Prog. Ser. A, 162(1-2):431-463, 2017.

[22] W. Slofstra. The set of quantum correlations is not closed. arXiv:1703.08618.

[23] B. S. Tsirelson. Quantum analogues of the Bell inequalities: The case of two spatially separated domains. Journal of Soviet Mathematics, 36:557-570, 1987.

[24] B. S. Tsirelson. Some results and problems on quantum Bell-type inequalities. Hadronic Journal Supplement, 8(4):329-345, 1993.

\section{A Omitted proofs}

\section{A.1 Proof of Lemma 2.4.}

Proof. Let $S$ denote the set in the right hand side of (17). Clearly, we have $\pi\left(\mathcal{E}_{n+m}\right) \subseteq S$. As $S$ is a compact convex set, for the converse inclusion it suffices to show that every extreme point of $S$ necessarily satisfies all the norm inequalities with equality. For this let $C \in \operatorname{ext}(S)$ and assume towards a contradiction that $\left\|u_{1}\right\|<1$. Select $\delta \in \mathbb{R}$ such that $\left\|u_{1}(1 \pm \delta)\right\| \leq 1$. Consider the $n \times m$ matrices $C^{+}, C^{-}$where $C_{i j}^{ \pm}=\left\langle u_{i}, v_{j}\right\rangle$, for all $i$ and $j \neq 1$, and $C_{1 j}^{ \pm}=\left\langle u_{1}(1 \pm \delta), v_{j}\right\rangle$, for all $j \in[m]$. Clearly, $C^{+}, C^{-} \in S$ and by definition $C=\left(C^{+}+C^{-}\right) / 2$. Thus, since $C^{+}, C^{-} \neq C$ we contradict the fact that $C \in \operatorname{ext}(S)$.

\section{A.2 Proof of Lemma 2.5.}

Proof. (i) This was shown already in the proof of Lemma 2.4.

(ii) Consider a $C$-system $\left\{u_{i}\right\}_{i=1}^{n},\left\{v_{j}\right\}_{j=1}^{m}$. We only show $\operatorname{span}\left(\left\{u_{i}\right\}_{i=1}^{n}\right) \subseteq \operatorname{span}\left(\left\{v_{j}\right\}_{j=1}^{m}\right)$, the other inclusion follows similarly. Towards a contradiction, say there exists some $i^{*} \in[n]$ such that $u_{i^{*}} \notin \operatorname{span}\left(\left\{v_{j}\right\}_{j=1}^{m}\right)$. Let $P$ be the orthogonal projector onto $\operatorname{span}\left(\left\{v_{j}\right\}_{j=1}^{m}\right)$. As $\left\|P u_{i^{*}}\right\|<\left\|u_{i^{*}}\right\| \leq 1$, the vectors $\left(\left\{u_{i}\right\}_{i=1}^{n} \backslash\left\{u_{i^{*}}\right\}\right) \cup\left\{P u_{i^{*}}\right\}$ and $\left\{v_{j}\right\}_{j=1}^{m}$ form a new $C$-system. Lastly, since $C \in \operatorname{ext}\left(\pi\left(\mathcal{E}_{n+m}\right)\right)$ and $\left\|P u_{i^{*}}\right\|<1$, this contradicts case $(i)$.

(iii) Consider a $C$-system $\left\{u_{i}\right\}_{i=1}^{n},\left\{v_{j}\right\}_{j=1}^{m}$. As $C \in \operatorname{ext}\left(\pi\left(\mathcal{E}_{n+m}\right)\right)$, by case $(i)$ we have that $\left\|u_{i}\right\|=\left\|v_{j}\right\|=1$, for all $i \in[n], j \in[m]$. This shows that $C \in \pi\left(\mathcal{E}_{n+m}\right)$ has at least one completion in $\mathcal{E}_{n+m}$. The next step is to show that $C$ it has a unique completion in $\mathcal{E}_{n+m}$. For this, let $\operatorname{Gram}\left(\left\{u_{i}^{\prime}\right\}_{i},\left\{v_{j}^{\prime}\right\}_{j}\right)$ and $\operatorname{Gram}\left(\left\{u_{i}^{\prime \prime}\right\}_{i},\left\{v_{j}^{\prime \prime}\right\}_{j}\right)$ be two elliptope completions. For $i \in[n], j \in[m]$ define $u_{i}=\frac{u_{i}^{\prime} \oplus u_{i}^{\prime \prime}}{\sqrt{2}}$ and $v_{j}=\frac{v_{j}^{\prime} \oplus v_{j}^{\prime \prime}}{\sqrt{2}}$ and note that they form a $C$-system. Thus, by case $(i i)$ we have that $\operatorname{span}\left(\left\{u_{i}\right\}_{i=1}^{n}\right)=\operatorname{span}\left(\left\{v_{j}\right\}_{j=1}^{m}\right)$. In particular, for all $i \in[n]$ there exist scalars $\left\{\lambda_{j}^{i}\right\}_{j=1}^{m}$ satisfying $u_{i}=\sum_{j=1}^{m} \lambda_{j}^{i} v_{j}$. By the definition of $u_{i}$ and 
$v_{j}$ this implies that $u_{i}^{\prime}=\sum_{j=1}^{m} \lambda_{j}^{i} v_{j}^{\prime}$ and $u_{i}^{\prime \prime}=\sum_{j=1}^{m} \lambda_{j}^{i} v_{j}^{\prime \prime}$. Then, for all $i, i^{\prime} \in[n]$ we get

$$
\left\langle u_{i}^{\prime}, u_{i^{\prime}}^{\prime}\right\rangle=\sum_{j=1}^{m} \lambda_{j}^{i}\left\langle v_{j}^{\prime}, u_{i^{\prime}}^{\prime}\right\rangle=\sum_{j=1}^{m} \lambda_{j}^{i}\left\langle v_{j}^{\prime \prime}, u_{i^{\prime}}^{\prime \prime}\right\rangle=\left\langle u_{i}^{\prime \prime}, u_{i^{\prime}}^{\prime \prime}\right\rangle .
$$

Analogously it follows that for all $j, j^{\prime} \in[m]$ we have $\left\langle v_{j}^{\prime}, v_{j^{\prime}}^{\prime}\right\rangle=\left\langle v_{j}^{\prime \prime}, v_{j^{\prime}}^{\prime \prime}\right\rangle$. Putting everything together we get that $\operatorname{Gram}\left(\left\{u_{i}^{\prime}\right\}_{i},\left\{v_{j}^{\prime}\right\}_{j}\right)$ and $\operatorname{Gram}\left(\left\{u_{i}^{\prime \prime}\right\}_{i},\left\{v_{j}^{\prime \prime}\right\}_{j}\right)$.

For any $C \in \operatorname{ext}\left(\pi\left(\mathcal{E}_{n+m}\right)\right)$ we denote by $E_{C}$ its unique elliptope completion. As the set of all completions of $C$ is a face of $\mathcal{E}_{n+m}$ it follows that $E_{C} \in \operatorname{ext}\left(\mathcal{E}_{n+m}\right)$ (here we use the fact that the only way for a single point to be a face is for the point itself to be extreme).

Lastly, we have already seen that $\operatorname{rank}\left(E_{C}\right)=\operatorname{rank}(A)=\operatorname{rank}(B)$. Clearly $\operatorname{rank}\left(E_{C}\right) \geq$ $\operatorname{rank}(C)$ and it remains to show that $r:=\operatorname{rank}(A) \leq \operatorname{rank}(C)$. Wlog assume that the first $r$ rows of $A$ are linearly independent. Then, $\sum_{i=1}^{r} \lambda_{i}\left\langle u_{i}, v_{j}\right\rangle=0$, for all $j \in[m]$ implies that $\sum_{i=1}^{r} \lambda_{i} u_{i}=0$ and thus $\lambda_{i}=0$, for all $i \in[r]$.

Acknowledgments. Both authors are supported in part by the Singapore National Research Foundation under NRF RF Award No. NRF-NRFF2013-13. 